Multiple Stars and Stellar Evolution

L. Kisseleva-Eggleton, P. P. Eggleton

January 10, 2011

Binary Star Evolution: Mass Loss, Accretion and Mergers Mykonos, Greece June 22, 2010 through June 25, 2010 
This document was prepared as an account of work sponsored by an agency of the United States government. Neither the United States government nor Lawrence Livermore National Security, LLC, nor any of their employees makes any warranty, expressed or implied, or assumes any legal liability or responsibility for the accuracy, completeness, or usefulness of any information, apparatus, product, or process disclosed, or represents that its use would not infringe privately owned rights. Reference herein to any specific commercial product, process, or service by trade name, trademark, manufacturer, or otherwise does not necessarily constitute or imply its endorsement, recommendation, or favoring by the United States government or Lawrence Livermore National Security, LLC. The views and opinions of authors expressed herein do not necessarily state or reflect those of the United States government or Lawrence Livermore National Security, LLC, and shall not be used for advertising or product endorsement purposes. 


\title{
Multiple Stars and Stellar Evolution
}

\author{
Ludmila Kisseleva-Eggleton, ${ }^{*}$ and Peter P. Eggleton ${ }^{\dagger}$ \\ * Expression College for the Digital Arts, Emeryville, CA, USA \\ ${ }^{\dagger}$ Lawrence Livermore National Laboratory, Livermore, CA, USA
}

\begin{abstract}
We discuss the incidence of multiplity, particularly among the bright and therefore relatively thoroughly examined stars, and note certain types of (a) binary stars that might be expected to merge, and (b) single stars with characteristics that suggest they may be former binaries that merged. Some Be stars and rapidly rotating red giants seem like possible merger products; and perhaps even some magnetic peculiar stars that are rapidly rotating.
\end{abstract}

Keywords: Bullshit

PACS: http://www.aip..org/pacs/index.html>

\section{INTRODUCTION}

According to Eggleton \& Tokovinin (2008), the population of 4558 stars brighter than Hipparcos mag. 6.00 (but excluding the Sun) divides into singles, binaries, triples etc. according to the first two columns of Table 1.

Naturally there must still remain some unrecognised components in these systems. Eggleton (2009) carried out an attempt at 'Multiple Star Population Synthesis', creating a theoretical population and then 'theoretically observing' it to see which subcomponents should be detectable and which not. One possible estimate (or rather ten, sampled randomly and averaged) is also shown in Table 1, in columns 3 to 6 . The 'raw' column is the multiplicity that was assigned, independently of whether the components were distinguishable or not, and the 'detectable' column is the multiplicity when only distinguishable systems or subsystems, i.e. those where there was a radial velocity amplitude over a certain threshhold, or where the angular separation was large enough in relation to the difference in magnitude, were counted. Naturally no such result can be considered definitive, because we can always increase the number of very faint and lowmass companions that would be below whatever criterion of detectability we apply. Note that planetary-mass objects were not included in either the observed or the estimated counts.

\section{EVOLUTIONARY PROCESSES}

Many of the orbits and sub-orbits in both the observed and the estimated distribution are sufficiently wide that no serious interaction between the components should have occurred. But a proportion are sufficiently close that some kind of binary interaction is expected. Roche-lobe overflow (RLOF) is the best-known such interaction, but there exist others, e.g. Kozai cycling combined with tidal friction (KCTF), in triple (or higher multiple) systems, or binary-enhanced stellar wind (BESW) before or instead of RLOF. 
TABLE 1. Observed and Estimated Multiplicities

\begin{tabular}{rccrcr}
\hline N & Observed & $\begin{array}{c}\text { Estimated } \\
\text { Raw }\end{array}$ & rms & $\begin{array}{c}\text { Estimated } \\
\text { Detectable }\end{array}$ & rms \\
\hline 1 & 2716 & 1459 & 63 & 2649 & 96 \\
2 & 1438 & 2179 & 111 & 1472 & 85 \\
3 & 285 & 517 & 37 & 302 & 37 \\
4 & 86 & 202 & 28 & 85 & 17 \\
5 & 20 & 101 & 10 & 29 & 5 \\
6 & 11 & 44 & 11 & 9 & 3 \\
7 & 2 & 30 & 13 & 3 & 3 \\
8 & 0 & 18 & 7 & 0 & 0 \\
av. & 1.53 & 2.04 & 0.03 & 1.55 & 0.02 \\
\hline
\end{tabular}

BESW may involve magnetic braking and tidal friction (MBTF). A further 'interaction', using the term loosely, is a supernova explosion (SNEX), which in most cases may simply eject the resulting neutron star from the system thus reducing its multiplicity. In the estimates of Table 1 however we have left such neutron stars inside their parent systems, for the purpose of keeping a census. 240 neutron stars (and 74 black holes) appeared in the 'raw' model, of which 43 (9) appeared as 'detectable' (through an RV orbit of the visible companion); in practice we expect that they would have been ejected and therefore become non-detectable. Arguably we should reduce the number of components in the model estimate of Table 1 , column 5 by $43+9$, but this is only a modest perturbation. In the observed set there is only one known NS companion, $\gamma$ Cas, although one or two others have been hypothesised.

The evolution of the components of the systems in the model distribution was taken from a pre-computed table of evolved single stars, according to their initial masses and ages. We tweaked the model to give the observed distribution over spectral type and luminosity, and over both period and multiplicity at a given spectral type; but the main guiding principle in the model was that it should be simple, and have as few adjustable constants as possible. In a first analysis the possible interactions were ignored, except that cases were noted in which a component would have actually filled its Roche lobe during this evolution. These amounted to $188 \pm 22$ in the model.

\section{SOME INTERESTING CASES}

An enjoyable but also problematic feature of our 'theoretical bright star catalogue' is its great diversity. There is almost an embarassment of riches. In one Monte Carlo realisation, for example, could one really have expected a system like \#161, where a core-helium-burning red giant is in a 38 day orbit with a binary of two F dwarfs in a 1.4 day sub-orbit? Well actually, yes: HD 216572 (V453 Cep) has such a giant in a 55 day orbit, the companion being a 1.2 day binary of two B9 dwarfs (Griffin \& Griffin 2009). How about an innocuous binary (\#770, 0.99 days) of two early B stars, but where four other components have become either a black hole or a neutron star? Perhaps even more surprisingly, an apparently single Cepheid (\#1211) of $8.5 M_{\odot}$ : its original 7 companions 
(original masses $9-24 M_{\odot}$ ) in an octuple system have all supernova'd.

We also generate quite a few systems with several white dwarfs in them, at least in the 'raw' state. Once we apply our detectability criteria most of the white dwarfs disappear. 'Snow White and the five dwarfs' was the best we could do (\#1930). Snow White was actually a post-AGB star, a highly luminous near-white-dwarf (cf HR 4049). One companion was in a 543 day orbit (cf 434 days for HR 4049). The others were older and fainter, typically 18th - 20th mag - and a few seconds or fractions of a second away. The most highly multiple WD system that we are aware of is triple (1704+481; Maxted et al. 2000), and that has no main-sequence or giant companion to move it into the the bright-star catalogue.

We must wonder, of course, whether such oddities are a result of an unrealistic multiplicity or mass-ratio distribution; but most of the detectable multiples generated are not very different from multiples seen. We need to have something of a bias towards equal masses in order to generate what is seen, and then it is not improbable that the one or two components that are detectable are the least massive, rather than the most massive, of the original components. We also need to have higher multiplicity at higher masses, because that is what is observed. The algorithm we used produced single to octuple systems, but no higher multiplicities.

The diversity is problematic, we find, because it becomes really difficult to categorise the systems. We had hoped that we would have found by now a reasonable set of say half-a-dozen bins ino which the great majority of theoretical (as well as actual) systems could be put, but we have tended to end up with something like one category per system, at least for the triples and upwards.

The kind of system that we are particularly interested in at present is one where a merger is expected to have happened. We believe that mergers are quite likely to be products of RLOF when this occurs in systems with mass ratios more extreme than 0.5 (or 2.0) - Nelson \& Eggleton (2001). We expect that several Be stars, specifically those which do not have a close binary companion, are merger remnants - although several other Be stars, such as $\gamma$ Cas mentioned above, may be remnants of RLOF in which a merger did not occur.

Consider the star HR 3574, which is a triple with components

$$
\left((V=4.69, \mathrm{~B} 5 \mathrm{~V}+? ; .9147 \mathrm{~d}, e=.13)+V=7.93 ; 2.560^{\prime \prime}\right)
$$

(Neubauer 1931). The primary is not a Be star. One would expect that it is rotating pretty rapidly if it corotates in its $0.9 \mathrm{~d}$ spectroscopic orbit, but this amounts to about $140 \mathrm{~km} / \mathrm{s}$ which is below what is normal for a Be star. The orbit is only single-lined, although Neubauer (1931) suspected a slight variation in the profile of one line that might have been due to a companion. The mass ratio might be as mild as $2: 1$, but we think this would still be enough to cause the RLOF to go out of control, leading to a merger when the B star is only halfway across the Main Sequence (MS) band. We would expect that the close pair would merge to a Be star, leaving a fairly wide binary behind.

The eclipsing SB2 $\alpha \mathrm{CrB}$, with parameters

$$
\left(\mathrm{B} 9.5 \mathrm{IV}+\mathrm{G} 2 \mathrm{~V} ; 2.58+0.92 M_{\odot} ; 17.36 \mathrm{~d}, e=.37\right)
$$


(Volkov 2005) has such a mass ratio that we think a merger is inevitable. The system will probably merge when the primary fills its Roche lobe shortly after it has crossed the Hertzsprung gap and reaches the bottom of the Giant Branch (GB). The merged remnant will presumably be a red giant, perhaps temporarily a supergiant like V838 Mon (Brown et al. 2002, Soker \& Tylenda 2003, Kamiński et al. 2009), and then probably settle down after $\sim 10^{5}$ yr to a red giant back at the bottom of the GB, but rotating somewhat faster than usual for a while. There exist rapidly-rotating red giants, FK Com stars, some of which may have originated this way although some are probably the merger products of the slow evolution of contact binaries (Webbink 1980).

We would nominate $\alpha$ Leo (HR 3982) and $\alpha$ Eri (HR 472) as possible prototypes for two different mechanisms for stellar spin-up. $\alpha$ Leo (B8IVn) is not a Be star, but with very rapid rotation $(317 \mathrm{~km} / \mathrm{s})$ it comes close. Gies et al. (2008) determined it to be an SB1 with period of $40.1 \mathrm{~d}$. The companion is probably a white dwarf. So $\alpha$ Leo is likely to be a post-RLOF system, where the former gainer is part-way through the process of spinning down, having presumably been spun up to Be velocity by the prior RLOF. There are several Be binaries, e.g. $\phi$ Per and $\gamma$ Cas, which are also post-RLOF but presumably at a slightly earlier stage, with much spin-down still to go.

$\alpha$ Eri (B3-4IIIe) has been found by adaptive optics (Kervella et al. 2008) to have a companion A star at $0.1-0.2^{\prime \prime}$; its position has varied measurably over two years. Kervella et al. estimate a period of $\sim 15 \mathrm{yr}$. Interferometric observations of $\alpha$ Eri show it to be highly oblate, with a projected axial ratio of 1.4, which suggests that it is rotating very close to breakup - the axial ratio should be 1.5 at breakup according to the simplest model. So $\alpha$ Eri is a reasonable prototype of a merged close binary.

$\mathrm{AB}$ Dor is a rapidly rotating $\mathrm{K}$ dwarf in a $\sim 12 \mathrm{yr}$ binary. Although far from being a $\mathrm{Be}$ star, and slightly fainter than our cutoff at 6th mag., we suspect it has a somewhat similar history to $\alpha$ Eri. Eggleton \& Kisseleva-Eggleton (2006) and Makarov \& Eggleton (2009) suggested that the present $12 \mathrm{yr}$ binary is the relic of a triple; the $\mathrm{K}$ dwarf is the merger remnant of an inner binary which had a short period $(\sim 3 \mathrm{~d})$ and a rather extreme mass ratio.

\section{MERGED REMNANTS IN FORMER TRIPLES}

Even if rapid rotation dies away fairly quickly, there is a possibility that a merged remnant will be recognisable in the following way. The triple system 1 Gem (HR 2134; Griffin \& Radford 1976, Heintz 1986)

$$
((\mathrm{G6III}+? ; 9.60 \mathrm{~d})+\mathrm{K} 0 \mathrm{III} ; 13.35 \mathrm{yr}, e=0.36),
$$

contains two red giants. We would ordinarily expect these to be of rather similar mass. But the inner binary must be well on the way to RLOF. We do not know the mass ratio in this inner system, but there is nothing inconsistent with the possibility that the mass ratio is small, say 0.5 , in which case the inner system is rather probably headed for a merger. After the merged remnant has settled back thermally it will probably still be a red giant, but it may now be nearly $50 \%$ more massive than the companion red giant.

A binary containing two red giants of considerably different masses might seem a monstrosity, but it is just what we should expect in some cases as a result of a merger. 
And there are examples - in fact two. One example is OW Gem (Griffin \& Duquennoy 1993):

$$
\left(\mathrm{G} 8 \mathrm{IIb}+\mathrm{F} 2 \mathrm{Ib}-\mathrm{II} ; 4.0+5.9 M_{\odot} ; 1259 \mathrm{~d}, e=.515\right),
$$

and the second is V643 Ori (Imbert 1987):

$$
\left(\mathrm{K} 7 \mathrm{III}+\mathrm{K} 2 \mathrm{III} ; 1.93+3.34 M_{\odot} ; 52.4 \mathrm{~d}, e=.014\right) .
$$

Some people may feel that the period of V643 Ori is improbably short for a former triple, but we have already mentioned HD 216572 with a similarly short outer period. In HD 216572 the inner binary has a sufficiently high mass ratio $(\sim 1)$ that we do not expect a merger, at least to start with. But the future evolution of this triple should be interesting nevertheless.

Our point is therefore that although it will be very difficult to recognise that a given single star is a merger remnant, it will be easier to recognised merger remnants in binaries, i.e. former triples. Among the bright stars, there are a handful (R. E. M. Griffin, p.c.) which have 'oversized secondaries', of which a good example is $\gamma$ Per (HR 915):

$$
\left(\mathrm{G} 8 \mathrm{II}-\mathrm{III}+\mathrm{A} 2 \mathrm{IV} ; 3.6+2.4 M_{\odot} ; 23+3.2 R_{\odot} ; 5350 \mathrm{~d}, e=0.79\right) .
$$

With a mass ratio of 1.5 , the secondary should be barely evolved from the ZAMS; and yet it is arguably more than half-way across the MS band. Another example is HD 126269 (R. E. M. Griffin p.c.).

\section{YET MORE MERGED REMNANTS?}

We would like to emphasise two recent papers of Bidelman $(2002,2005)$. In the earlier paper, he suggests that the classic Ap magnetic stars may be merger remnants, and points in particular to HR Lup (HR 5624, B8IVpSi) which rotates in $0.877 \mathrm{~d}$. In the second paper he identifies many radioactive species in the notoriously peculiar HD 101065 (Przybylski's star) - not only Tc and Pm, but also several trans-uranic species.

The case for believing that the chemical peculiarities of magnetic Ap stars are due to nuclear reactions went out of fashion in the late 60s, to be replaced by the theory that diffusive separation is the basic cause. Obviously this will not be the cause of radioactive species, however. What may be the cause is spallation, with particles above the photosphere being accelerated by the very strong magnetic fields to cosmic-ray energies.

The interaction of rotation with magnetic field is no doubt complex, but it does not seem improbable to me that very rapid rotation encourages dynamo activity in the convective core of a B star, just as it does in the convective envelope of a $\mathrm{G} / \mathrm{K} / \mathrm{M}$ star; but the timescale may be much slower. Perhaps very rapid rotation and Be characteristics are followed, after a fairly short time, by magnetic field production and somewhat less rapid rotation: HR Lup being a later stage of $\alpha$ Eri. Once a strong magnetic field is established - perhaps episodically, with a timescale of thousands of years - the star would spin down much more rapidly, at least as long as there is a trace of stellar wind, so that the typical Bp star will be slowly rotating for most of its life. 
In summary, we believe some Be stars, and perhaps also some magnetic Ap stars, are merger products, and that many more merger products must exist even in the rather small but well-studied set of naked-eye stars. Our hope is that the D jehuty project at Lawrence Livermore National Laboratory, a project that models stars in 3D, will throw light on both the merger process and the interaction of magnetic field with rotation.

\section{ACKNOWLEDGMENTS}

This study has been carried out under the auspices of the U.S. Department of Energy, National Nuclear Security Administration, under Contract DE-AC52-07NA27344. I gratefully acknowledge the help of the Centre des Données Stellaires (Strasbourg), and of the Astronomical Data System.

\section{REFERENCES}

Bidelman, W. P. (2005) ASPConf.Ser, 336, 309

Bidelman, W. P. (2002) Obs, 122, 343

Brown, N. J., Waagen, E. O., Scovil, C., Nelson, P., Oksanen, A., Solonen, J. \& Price, A. (2002) IAU Circ 7785

Eggleton, P. P. (2009) MNRAS, 399, 1471

Eggleton, P. P. \& Tokovinin, A. A. (2008) MNRAS, 389, 869

Eggleton, P. P. \& Kisseleva-Eggleton, L. (2006) Ap\&SS, 304, 75

- Gies, D. R., Dieterich, S., Richardson, N. D., Riedel, A. R., Team, B. L., McAlister, H. A., Bagnuolo, W. G., Jr., Grundstrom, E. D., Stefl, S., Rivinius, Th. \& Baade, D. (2008) ApJ, 682, L117

Griffin, R. F. \& Duquennoy, A. (1993) Obs, 113, 53

Griffin, R. E. M. \& Griffin, R. F. (2009) MNRAS, 394, 1393

Griffin, R. E. M., Hünsch, M., Marshall, K. P., Griffin, R. F. \& Schröder, K.-P. (1993) A\&A, 274, 225

Griffin, R. F. \& Radford, G. A. (1976) Obs, 96, 188

Heintz, W. D. (1986) A\&AS, 65, 411

Imbert, M. (1987) A\&AS, 71, 69

Kamiński, T., Schmidt, M., Tylenda, R., Konacki, M. \& Gromadzki, M. (2009) ApJS, 182, 33

Kervella, P., Domiciano de Souza, A. \& Bendjoya, Ph. (2008) A\&A, 484, L13

Makarov, V. V. \& Eggleton, P. P. (2009) ApJ, 703, 1760

Maxted, P. F. L., Marsh, T. R., Moran, C. K. J. \& Han, Z. (2000) MNRAS, 314, 334

Nelson, C. A. \& Eggleton, P. P (2001) ApJ, 552, 664

Neubauer, F. J. (1931) Lick Obs Bul, 15, 104

Soker, N. \& Tylenda, R. (2003) ApJ, 582, L105

Volkov, I. M. (2005) Ap\&SS, 296, 105

Webbink, R. F. (1980) in Close Binary Systems: Observations and Interpretations eds Plavec, M. J., Popper, D. M. \& Ulrich, R. K., IAU Symp. 88, p127 